\title{
An HR-EBSD and computational crystal plasticity investigation of microstructural stress distributions and fatigue hotspots in polycrystalline copper
}

\author{
V.V.C. Wan ${ }^{1 *}$, M.A. Cuddihy ${ }^{1}$, J. Jiang ${ }^{1}$, D.W. MacLachlan ${ }^{2}$, F.P.E. Dunne ${ }^{1}$ \\ ${ }^{1}$ Department of Materials, Imperial College, London, SW7 2AZ, UK \\ ${ }^{2}$ Rolls-Royce plc, PO Box 31, Derby, DE24 8BJ, UK \\ * Corresponding author: E-mail address: v.wan12@imperial.ac.uk
}

\begin{abstract}
High resolution EBSD studies on a deformed copper polycrystal have been carried out to quantify the microstructural residual stress distributions, and those of stress state including triaxiality of importance in defect nucleation studies. Crystal plasticity analysis of a representative, similarly textured, model polycrystal has been carried out showing that the experimental distributions of microstructural residual stress components, effective stress, hydrostatic stress and stress triaxiality are well captured.
\end{abstract}

The crystal model enables point-wise microstructural Schmid factors to be calculated both globally (ie with respect to the macroscopic remote loading) and locally from full knowledge of the grain-level stress state. Significant differences are demonstrated such that global Schmid analysis tends to overestimate slip activity and the frequency of high Schmid factors, indicating that the local microstructural heterogeneity is significant and caution is necessary in interpreting polycrystal behaviour using global Schmid factors.

A stored energy criterion for fatigue crack nucleation indicates that preferential sites for fatigue crack nucleation are local to grain boundaries (as opposed to triple junctions), and that hard-soft grain interfaces where high GND densities develop are preferable.

Keywords: Schmid factor, fatigue crack nucleation, crystal plasticity 


\section{Introduction}

Advanced engineering alloys used in industrial components are in high demand and must be designed for optimum resistance to creep, fatigue and environmental degradation. To further progress and develop the next generation of alloys, a greater understanding of polycrystalline materials at the microstructural scale must be addressed. It is well accepted that crystallography, morphology and interface constraints contribute to the heterogeneity in local stress, strain and dislocation density witnessed across grains [1-4], and much of the heterogeneity arises from anisotropic slip activity. Dislocation slip on a given crystal system is initiated according to Schmid's rule when the resolved (Peierls) shear stress on any slip system exceeds the intrinsic strength of the system, often described by a critical resolved shear stress (CRSS). This leads to slip activation, the motion of dislocations, and potentially dislocation accumulation and hardening. This often promotes the activation of other slip systems, including cross-slip, resulting in multislip but in all active slip systems, the local Peierls stress must exceed the intrinsic slip strength. Other approaches to understand generalised slip include the Taylor method [5], which assumes all grains undergo the same shape change as the entire polycrystal, driving the need for a minimum five independent slip systems to be activated simultaneously in order to maintain geometric compatibility. However, with recent developments of strain mapping techniques such as sub-micron Digital Image Correlation (DIC) [6] and crystal plasticity finite element modelling (CPFEM) [7-9], localised strain heterogeneity in polycrystals is observed which cannot be determined from macroscopic considerations, and the assumption of homogeneous deformation is simply wrong. In advanced CPFEM models [7-9], Schmid factor is utilised in constitutive formulations to determine the resolved shear stress on a given crystal system. When the slip strength is reached in a given slip system, slip is allowed to occur. This does allow multiple slip systems to be activated as long as the resolved shear stress is larger than the critical value. This is in fact different from Schmid law as originally and simply posed, which assumed that only single slip is activated for all grains within polycrystals. High resolution-EBSD studies have also shown $[10,11]$ non-homogeneous localisation of stresses in terms of both magnitude and direction at the grain scale.

Schmid factors have been extensively referenced in many studies [12-18]. Sriram et al. [12] have shown that dislocation pinning events may not be affected by Schmid factor differences. Dislocation interactions with grain boundaries (GB) have been an active topic of research and Kacher et al. [14] discuss some local sensitive criteria for slip transmission across a GB based on geometry, resolved shear stress, Schmid factor, burgers vector and interfacial energy. Some authors have reviewed [16] slip transfer parameters weighted using Schmid factors specifically along GBs, considering both local stress and macro stress in neighbouring grains. Quantifying Schmid factors across polycrystals also identifies soft and hard grains for slip giving a methodology used by Wang et al. [15] to investigate the relationships between activated dislocation slip and twinning systems. Other authors [17] 
considered dislocation channel and GB interactions and argued that the lower the Schmid factor in a grain adjacent to a discontinuous channel, the higher the strain in the channel impinging on the grain. However, many of the studies described here utilise the global stress tensor to quantify the local Schmid factor. Lim et al. [18] showed in their combined experimental and crystal plasticity modelling studies on Ta oligocrystals that under high strain, grains with low (global) Schmid factors or apparently unfavourably orientated for slip often presented large deformations which were also experimentally observed. This contradicts the understanding of Schmid law where favourably orientated grains should show greater propensity for slip. Hence as described by the authors [18], the use of global stress state Schmid factor can be problematic for the determination of local slip activation.

Due to the recognition of the importance of Schmid factors with respect to dislocation slip, some authors [19-24] have utilised these key microstructural quantities to understand local micromechanical behaviour for crack nucleation. Birosca et al. [19] utilised the Schmid factor, Taylor factor and GND density to address the effect of local texture on crack propagation path in $\mathrm{Ni}$ superalloy and found that secondary cracks are more prone to nucleate on triple junctions between soft and hard orientated grains, and the Taylor factor parameter was found not to give any direct indication of dislocation activity within a grain. Mesoscale modelling [20] with quantified Schmid factor in a polycrystal also concluded micro-crack initiation near defects is highly dependent on adjacent grain orientations. Other damage nucleation studies [21] assessed a fracture initiation parameter considering only the Schmid factor of highly stressed twinning systems, to support four of the twelve hypotheses on damage nucleation. Though these studies used Schmid parameters to link with failure, experimental studies by Risbet et al. [22] have shown local damage initiation of fatigue extrusion heights in Ni-superalloy have both high and low Schmid factors. More recent modelling work by Phung et al. [23] used the Schmid criterion to predict preferential sites of persistent slip marking (PSM) only to conclude that the prediction of the two different types of PSMs they considered could not be achieved. Understanding local slip behaviour in micro-volumes [25] or macrozones in $\mathrm{Ti} 6 \mathrm{Al} 4 \mathrm{~V}$ [26] from macroscopic stress states also remains problematic. To date, local stress effects and their role in Schmid factor determination have not been thoroughly investigated. In addition, questions remain whether the Schmid criterion alone is sufficient to capture sites for defect nucleation compared to other fundamental approaches such as energy based methodologies [7,27] which have shown some success for fatigue crack nucleation.

This paper presents an assessment of Schmid factors in polycrystal deformation slip and fatigue crack nucleation in which the classical Schmid calculation is carried out based on macro-level stress state, together with the local calculation where full detail of grain-level stress heterogeneity is accounted for. High resolution EBSD measurements from Jiang et al. [10] are used to assess local stress states after 
large deformation in oxygen-free high conductivity (OFHC) copper polycrystals. A corresponding polycrystal plasticity representative volume element (RVE) model is developed for quantitative study of deformation history and local, grain-level quantities, including Schmid factors, stress components, and stress states (including hydrostatic stress and triaxiality) which are potentially important in fatigue nucleation studies. In particular, fatigue crack nucleation hotpots are identified using a stored energy criterion which is assessed in the context of local microstructural features and the grain-level stressstate quantities.

\section{Microstructurally-sensitive stress quantities and experimental and computational methodology}

In this section, a brief description and definitions of the microstructurally-sensitive quantities addressed are given, together with the overall research methodology in which we carry out measurement of key microstructure-sensitive quantities using HR-EBSD, and crystal plasticity modelling, and investigate statistical distributions of such quantities within representative polycrystals. Microstructure-sensitive quantities examined include:

- Local and global Schmid factors

- Local and global resolved shear stresses

- Local stress components and stress states (including hydrostatic and effective stresses and stress triaxiality)

A schematic of a single crystal rod under tension is shown in Fig. 1. The applied tension follows the $y$-direction of the rod sample, where the corresponding active slip plane normal $\tilde{n}$ lies at angle $\phi$ from the load vector, and the slip direction $\tilde{s}$ at $\lambda$ to the applied load direction, as detailed in textbooks [28].

(a)

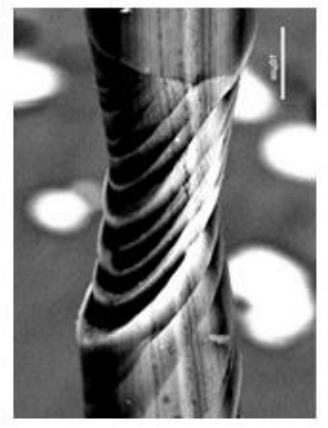

(b)

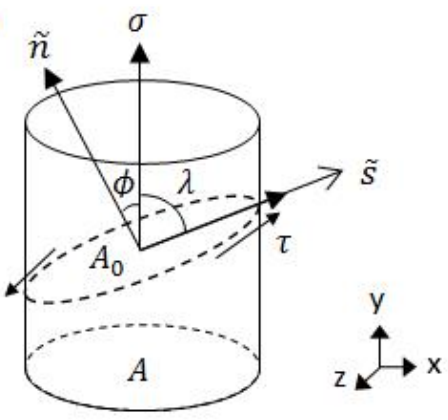

Fig. 1 - (a) SEM image of discrete slip planes under deformation in a single crystal, and (b) schematic annotation of single crystal slip in a rod subjected to a uniaxial y-direction loading

For the single crystal, single slip conditions shown, the Schmid factor (SF) is simply expressed as

$\mathrm{SF}=\cos \emptyset \cos \lambda$

The resolved shear stress on the slip system shown under uniaxial conditions is then 
$\tau=\sigma \cos \phi \cos \lambda$

which may be re-written as

$\tau_{r}=\sigma(\tilde{s} . \tilde{l})(\tilde{n} . \tilde{l})$

where $\tilde{l}$ is the uniaxial loading direction vector, $\tilde{s}$ the slip direction and $\tilde{n}$ the slip normal. From Eq.3 and Schmid factor $(\tilde{s} . \tilde{l})(\tilde{n} . \tilde{l})$, once the resolved shear stress $\tau_{r}$ reaches the critical resolved shear stress $\tau_{c}$ (CRSS), slip initiation takes place according to Schmid's Law. More generally, multiple slip systems within a given grain may potentially become active when the resolved shear stress equates to the CRSS value. Hence a high Schmid factor value would indicate the grain is favourably oriented for slip and would appear to be a 'soft' grain; meanwhile a low Schmid factor value would indicate the grain to be unfavourably oriented for slip and appear to be a 'hard'.

In a polycrystal containing $\eta$ grains, provided the crystallographic orientation of each grain is known, the resolved shear stress on each slip system in each grain may be determined, and often, this is done by assuming the remote, applied stress, $\sigma$ remains unchanged in magnitude and direction at the individual grain level. This we term the remote, or macro-level resolved shear stress.

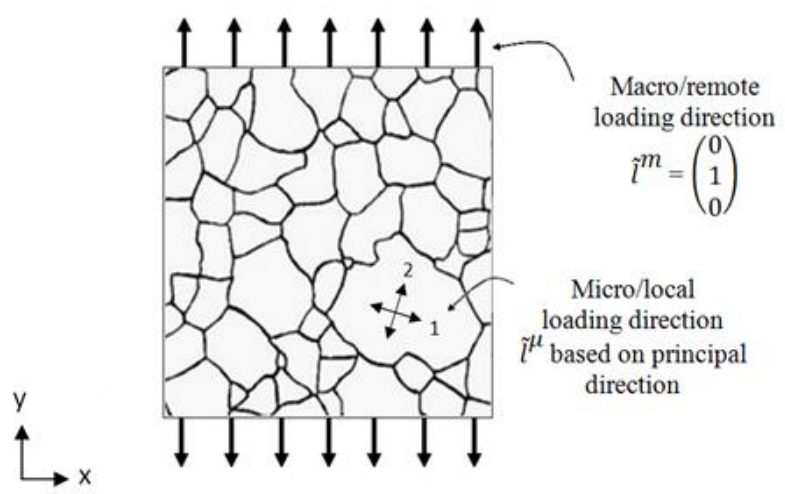

Fig. 2 - Schematic diagram of a polycrystal sample under uniaxial tensile loading in the $\mathrm{Y}$ direction showing the local load direction $\tilde{l}^{\mu}$ based on the maximum principal stress (1) direction and the macro-load direction, $\tilde{l}^{m}$.

An illustration of the remote loading direction is shown in Fig. 2 where the remote loading direction is simply the direction of the external force applied, shown as $\tilde{l}^{m}$. However, in reality, the constraint developed by grain-to-grain interactions with differing crystallographic orientation and hence elastic moduli, together with the anisotropy of slip, contrive to cause multiaxial and heterogeneous stress states to be developed at the grain level which are potentially quite different to those generated by the remote loading. As a consequence, not only is the magnitude of the local stress different to the macrolevel, but the directions of the principal stresses may well not align with the remote loading direction either. In order to determine slip activity, therefore, it is necessary to calculate the local resolved shear 
stress based on the local multiaxial stress state. In order to investigate systematically the differences resulting from the macro-level (indicated with $m$ ) and local (indicated $\mu$ ) approaches, the corresponding Schmid factors (SF) are defined by

$\mathrm{SF}^{\mathrm{m}}=\left(\tilde{s}^{\alpha, \eta} \cdot \tilde{l}^{m}\right)\left(\tilde{n}^{\alpha, \eta} \cdot \tilde{l}^{m}\right)$

$\mathrm{SF}^{\mu}=\left(\tilde{s}^{\alpha, \eta} \cdot \tilde{l}^{\mu}\right)\left(\tilde{n}^{\alpha, \eta} \cdot \tilde{l}^{\mu}\right)$

where the term $\alpha$ corresponds to the crystal slip number, in this case consisting of $12\langle 110\rangle$ [1 11 l] fcc slip directions and planes. The true resolved shear stress may be rigorously determined from $\tau=$ $(\tilde{\sigma} \tilde{n}) . \tilde{s}$ and for the purpose of our study local $\left(\tau^{\mu}\right)$ and global $\left(\tau^{m}\right)$ resolved shear stresses are introduced and can also be evaluated from

$\tau^{m}=\sigma_{l} \mathrm{SF}^{\mathrm{m}}$

$\tau^{\mu}=\sigma_{\operatorname{maxps}} \mathrm{SF}^{\mu}$

where $\sigma_{l}$ is the macro-level applied stress and $\sigma_{\text {maxps }}$ is chosen to be the local, grain-level maximum principal stress and Eq. 8 provides the general comparison to be made between the macro and local Schmid approaches.

The other key local, grain-level quantities investigated include the effective stress, the hydrostatic stress and the stress triaxiality ratio because of their relevance to defect nucleation studies. In terms of principal stresses, the effective stress is expressed as

$\sigma_{e}=\frac{1}{\sqrt{2}}\left[\left(\sigma_{1}-\sigma_{2}\right)^{2}+\left(\sigma_{2}-\sigma_{3}\right)^{2}+\left(\sigma_{3}-\sigma_{1}\right)^{2}\right]^{1 / 2}$

In the experimental measurements considered later, the grain-level stresses have been obtained from HR-EBSD studies in which the stress components may be determined but only with respect to some point at which the absolute stress is known. The latter is generally different for each grain (or indeed unknown), so to overcome this problem [29], we instead examine stress differences determined as the deviation of the local stress from the grain average. Hence, the effective stress differences $\Delta \sigma_{e}^{\mathrm{n}}$ at each spatial local point (n) across a microstructure is determined using

$\Delta \sigma_{e}^{\mathrm{n}}=\sigma_{e}^{\mathrm{n}}-\sigma_{e}^{\mathrm{n}, \text { mean }}$

where $\sigma_{e}^{\mathrm{n}, \text { mean }}$ is the mean effective stress within the grain within which the local point (n) lies.

The hydrostatic stress is determined from

$\sigma_{H}=\frac{1}{3}\left(\sigma_{11}+\sigma_{22}+\sigma_{33}\right)$ 
where similarly the hydrostatic stress differences within each individual grain are given by

$\Delta \sigma_{H}^{\mathrm{n}}=\sigma_{H}^{\mathrm{n}}-\sigma_{H}^{\mathrm{n}, \text { mean }}$

Stress triaxiality is a commonly utilised parameter in quantifying stress state dependence of defect nucleation and ductile failure. For example, studies in austenitic steel show that effective plastic strain decreases exponentially with increasing stress triaxiality ratio at fracture [30]. The triaxiality ratio is defined in terms of stress differences by

$R_{\text {Triaxiality }}^{n}=\Delta \sigma_{H}^{\mathrm{n}} / \Delta \sigma_{e}^{\mathrm{n}}$.

Detailed quantitative and qualitative studies are presented in both experimentally tested copper polycrystals along with representative 3D polycrystal plasticity RVE models, and for both, the statistical distributions of the key microstructure-sensitive quantities introduced above are assessed. In addition, the identification of microstructural 'hotspots' for fatigue crack nucleation based on a stored energy method are identified within the representative polycrystal model, and related, where possible, to key microstructural features as well as the microstructure-sensitive quantities already discussed. In the next section, the experimental EBSD investigations are presented.

\section{Microstructure sensitivity in polycrystalline copper using HR-EBSD}

The cross-correlation-based HR-EBSD technique is used to map the intragranular residual stresses in an OFHC copper polycrystal after 6\% uniaxial strain deformation. The full detail of the HR-EBSD methodology for residual stress determination may be found in [31-33]. A segment of the (OFHC) copper polycrystal is identified and illustrated in Fig. 3.

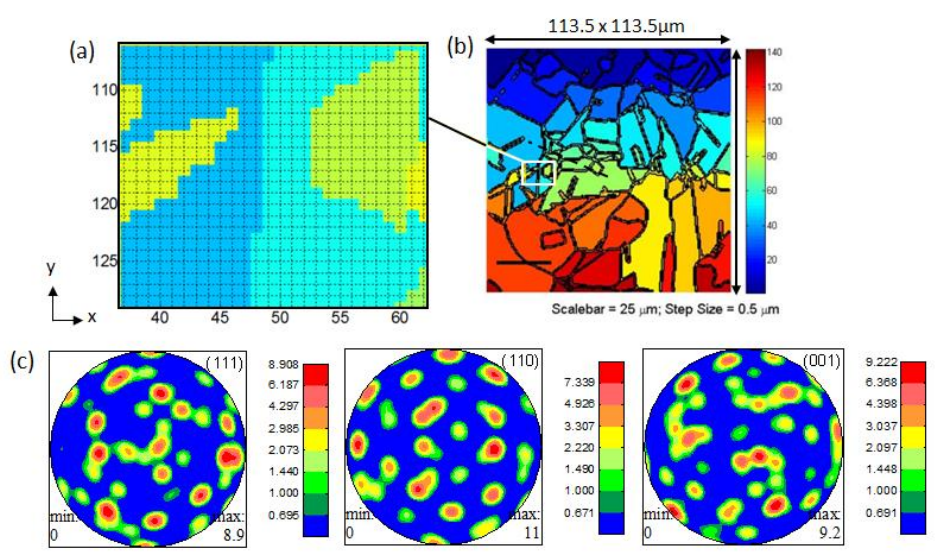

Fig. 3 - Microstructural field of interest in Cu polycrystal showing (a) representative grid where each crossingline represents a single grid point for data extraction, (b) EBSD scan with 51,529 data points with grain boundary mapping and grain ID legend, and (c) pole figures representative of the mapped field 
The $113.5 \times 113.5 \mu \mathrm{m}$ microstructure Fig. 3(b), contains a $227 \times 227$ grid for which each cross-section point from $\mathrm{n}=1$ to 51529 contains full details of local residual stress components after $6 \%$ deformation obtained from HR-EBSD. A close up of a region of the grid is shown in Fig. 3(a) and has been taken from [10]. The pole figure plots in Fig. 3(c) of the microstructure region of interest show weak texture containing 142 grains (misorientation tolerance of 10 degrees) with average grain size found to be about $10 \mu \mathrm{m}$.
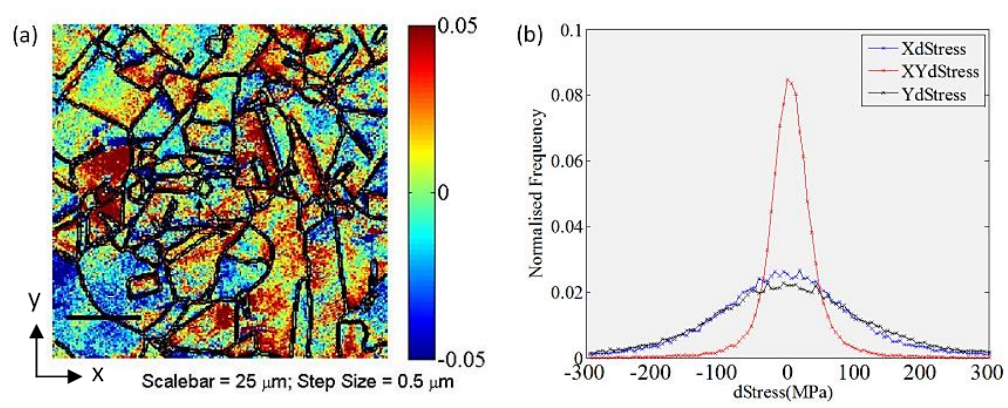

Fig. 4 - Cu polycrystal after 6\% deformation (in y-diection) and unloaded showing (a) experimental in-plane residual shear stresses from HR-EBSD (legend in GPa) and (b) histograms of stress component differences calculated pointwise.

After $6 \%$ uniaxial deformation along the y-direction, the full field distribution of normalised residual shear stresses with respect to individual grain reference points is shown in Fig. 4(a). Note that the residual stress determined here is the intragranular stress and further elaboration of the technique can be found in a recent review [4]. Large heterogeneity is observed together with strong localisation of residual shear stress across the microstructure and within individual grains. Distribution plots of three normalised in-plane stress components are shown in Fig. 4(b). The $\mathrm{xx}$ and yy stress difference components follow a normal distribution curve with a much lower frequency of direct peak stress than for the peak shear stress. Further, most of the direct stress differences lie within a range of $+/$ 300MPa stress. Using Eq. 10 and Eq. 12, the effective and hydrostatic stress differences across the microstructure are plotted in Fig. 5(a). Both the stress differences lie within +/- 400 MPa but the effective stress (difference) is slightly skewed to a mean value of $-120 \mathrm{MPa}$. As the effective stress is always positive, from Eq. 10 this would indicate the mean residual effective stress within a grain has a magnitude difference of $120 \mathrm{MPa}$ across individual grains. The distribution of the residual stress stored within the microstructure and its variation makes clear the level of inhomogeneity present.

The maximum principal stresses and directions are calculated by obtaining the eigenvalues of the residual stress tensors. The local principal direction is compared with the remote loading direction (y axis) and exposes the distribution of deviation shown in Fig. 5(b) with a standard deviation of $30^{\circ}$. This implies that due to the local influence of morphology and crystallography, not all local grains are deformed under the same uniaxial loading state and there are significant deviations in the local 
loading direction. In the next section, a representative $\mathrm{Cu}$ polycrystal model is presented to investigate the development of the local stress states. Firstly, microstructure-level stresses and their polycrystal distributions are assessed against the experimental measurements presented above. This is then followed by an assessment of local versus global Schmid analysis for polycrystal samples under loading.
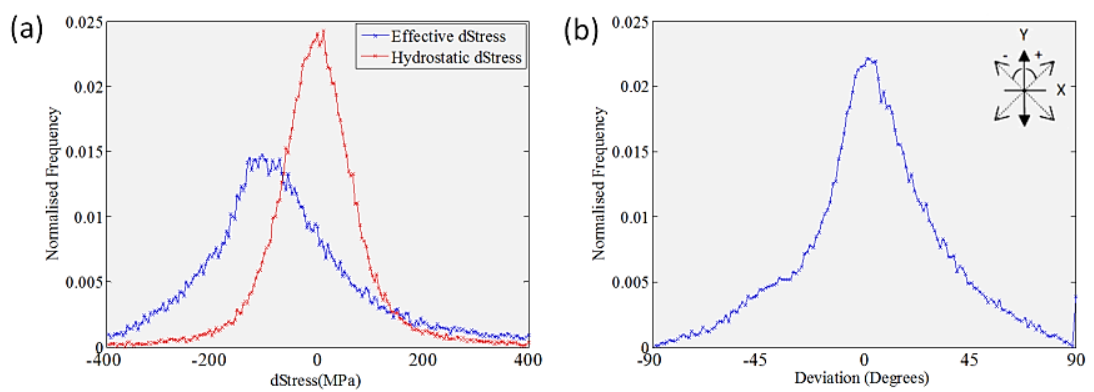

Fig. 5 - (a) Hydrostatic and effective stress differences across the microstructural region of interest and (b) distribution of angular deviation between the remote load direction and the local principal stress direction (in degrees)

\section{Assessment of microstructure-sensitive stresses, stress states and Schmid factors by crystal plasticity finite element modelling}

3D crystal plasticity modelling is used to model and investigate the behaviour of polycrystalline copper using a representative volume element (RVE). The objective is to investigate local grain-level behaviour and in particular, the microstructural quantities assessed experimentally and described earlier. The model $\mathrm{Cu}$ polycrystal is deformed to $6 \%$ uniaxial straining, followed by unloading to leave a residual stress state, and generic comparisons are therefore possible between the experimental observations for the polycrystal shown in Fig. 3 and the crystal model results.

The finite element RVE comprises 100 uniform hexagonal-shaped grains with three elements through the sample depth. Uniaxial straining to $6 \%$ is imposed followed by complete unloading to replicate the experimental procedure. The RVE copper polycrystal measures $180 \times 200 \mu \mathrm{m}$ with average grain size $20 \mu \mathrm{m}$ and is shown in Fig. 6. Twenty-noded brick finite elements with reduced integration (C3D20R) are employed. All results are extracted from integration points on the free front surface of the model with spacing of $0.5 \mu \mathrm{m}$ in order to replicate the experimental grid size shown in Fig. 3(a).

Pole figure plots to describe the RVE copper microstructure texture are shown in Fig. 6(a) which may be compared directly with the experimentally determined texture shown in Fig. 3(b). With respect to the experimental polycrystal microstructure assessed, due to low stacking fault energy, a considerable number of annealing twins $(\Sigma 3)$ are present in the microstructure which may be seen in Fig. 3. The twins are not captured in the RVE model because of the consistent grain morphology adopted, but the experimental texture is represented. Hexagonal-shaped grains are used within the RVE model to 
introduce local features such as triple junctions and differing angles of grain boundaries with respect to the loading direction.
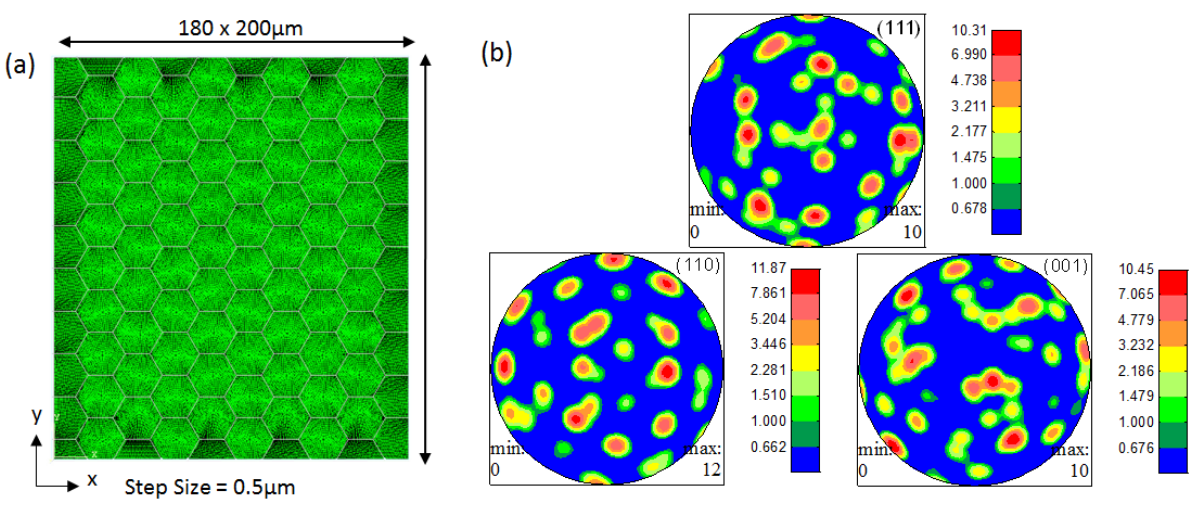

Fig. 6 - (a) Example surface mesh of the RVE model, and (b) pole figure plots of the RVE model texture

The kinematic decomposition of the deformation gradient $\mathbf{F}$ can be expressed in terms of the elastic $\mathbf{F}^{\mathrm{e}}$ and plastic $\mathbf{F}^{\mathrm{p}}$ tensors as described in [34] such that

$\mathbf{F}=\mathbf{F}^{\mathrm{e}} \mathbf{F}^{\mathrm{p}}$

The crystal plasticity model written in a UEL subroutine and integrated within the abaqus finite element code, expresses the plastic velocity gradient $\mathbf{L}^{\mathbf{p}}$ in terms of the summation of the contributions from all active slip systems, each with given rate $\dot{\gamma}^{\mathbf{i}}$, system normal $\mathbf{n}^{\mathbf{i}}$, and slip direction $\mathbf{s}^{\mathbf{i}}$ such that

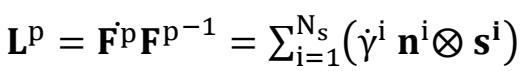

where $\mathrm{N}_{\mathrm{s}}$ indicates the summation of all of the twelve potentially active fcc slip systems, such that the accumulated slip rate $\dot{p}$ arising from the summation of contributions from all active slip systems is determined by

$\dot{p}=\left(\frac{2}{3} \mathbf{L}^{\mathrm{p}}: \mathbf{L}^{\mathrm{p}}\right)^{1 / 2}$.

The crystal plasticity framework is similar to that described in previous works $[7,35,36]$ and explicit coupling of statistically stored dislocation (SSD) and geometrically stored dislocation (GND) density, both leading to hardening, are included. The crystal plasticity model is integrated within the finite element-based solver ABAQUS using a user element subroutine (UEL) which allows local plastic strain gradients to be captured. The slip rate is given by

$\dot{\gamma}^{\eta}=\rho_{S S D} v\left(b^{\eta}\right)^{2} e^{\left(-\Delta H^{\eta} / k T\right)} \sinh \left(\frac{\left(\tau_{r}^{\eta}-\tau_{c}{ }^{\eta}\right) \gamma_{0}\left(b^{\eta}\right)^{2}}{k T \sqrt{\rho_{S S D}+\rho_{G N D}}}\right)$

where $v$ is the energy barrier jump frequency, $b$ the Burger's vector magnitude for a given slip system $\eta, \Delta H$ the Helmholtz free energy, $k$ the Boltzmann constant, $T$ the temperature, $\tau_{r}$ the resolved shear stress (determined using Eq. (3)) and $\rho$ indicates dislocation density of the either SSD or GND type. 
Schmid's law is used to determine slip activation in a crystal when the resolved shear stress in a particular slip system reaches the critical resolved shear stress. The latter is allowed to evolve based on the evolution of dislocation density to capture the hardening effects as outlined in [7] and is determined by Taylor type hardening

$\tau_{c}{ }^{\eta}=\tau_{c_{o}}{ }^{\eta}+\mu G b \sqrt{\rho_{S S D}+\rho_{G N D}}$.

The representative model developed is calibrated to match the experimental macroscopic stress strain response for the experimentally tested OFHC copper polycrystal [10], and the resulting experimental and CPFE macroscopic stress strain response is shown on Fig. 7(a) using the slip rule parameters in Table 1. The boundary and loading (and unloading) conditions imposed are also shown in Fig. 7(b) and (c).

(a)

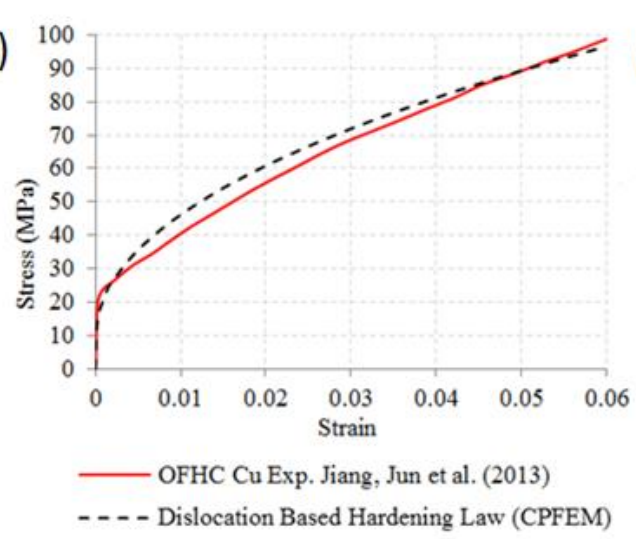

(c)

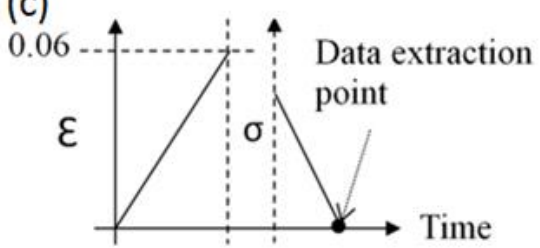

(b)

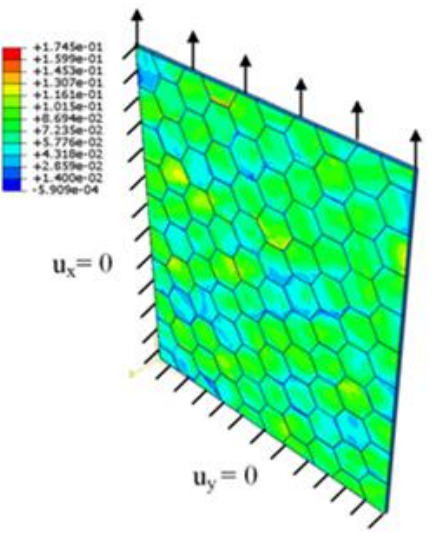

(d)

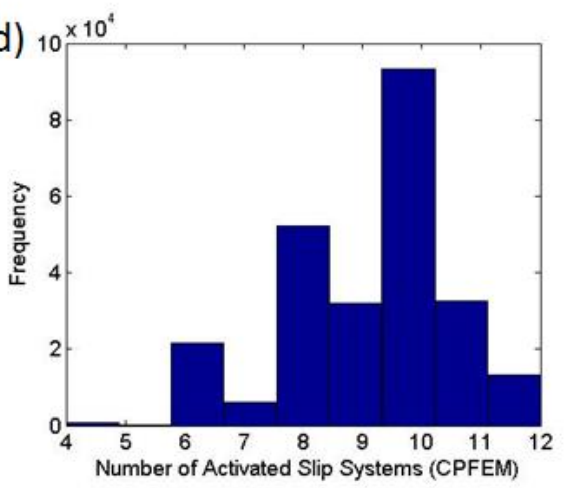

Fig. 7 - (a) Experimental and crystal model tensile stress-strain response up to 6\% strain showing considerable material hardening, (b) schematic of loading direction and boundary conditions with accumulated slip distribution after $6 \%$ deformation in the unloaded state, (c) loading history and data extraction point after $6 \%$ deformation in the unloaded state to replicate the experimental conditions of measurement, (d) number of active slip systems across the crystal model shown in Fig. 7(b). 
Table 1 - Material properties for the OFHC copper polycrystal calibrated in the CPFE model

\begin{tabular}{cc}
\hline$b$ & $3.5 \times 10^{-10} \mathrm{~m}$ \\
$\tau_{c_{o}}$ & $3.5 \mathrm{MPa}$ \\
$\rho_{s s D}$ & $0.5 \times 10^{10} \mathrm{~m}^{-2}$ \\
$\Delta H$ & $4.91 \times 10^{-20} \mathrm{~J}$ \\
$\gamma_{o}$ & $6.0 \times 10^{-4}$ \\
$k$ & $1.381 \times 10^{-23} \mathrm{JK}^{-1}$ \\
$v$ & $1.0 \times 10^{11} \mathrm{~s}^{-1}$ \\
\hline
\end{tabular}

In addition, the distribution of the number of active slip systems in the polycrystal model contributing to the accumulated slip distribution in Fig. 7(b) is plotted in Fig. 7(d) showing that multislip occurs commonly.

(a)

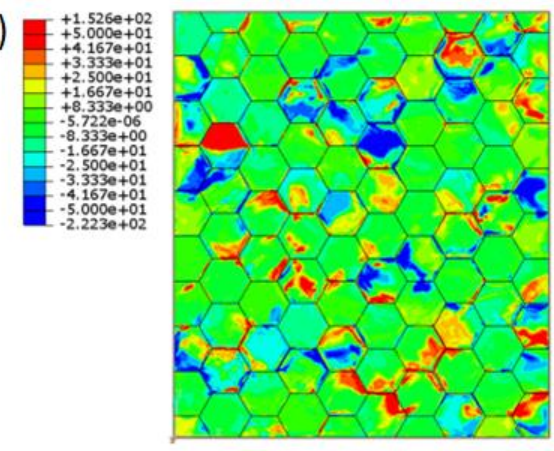

(b)

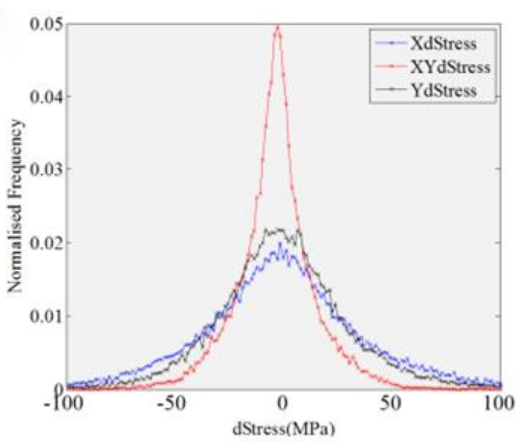

Fig. 8 - (a) Field variation of shear stress difference and (b) frequency distribution plots of stress component differences for the model crystal plasticity polycrystal

After 6\% applied deformation, and following the unloading shown in Fig. 7(c) such that the sample material is in a fully relaxed and unloaded state, the direct and shear stress component differences are extracted in the same way as that for the experimental residual stresses, and are shown in Fig. 8 in which both the field variation of shear stress difference is shown together with the frequency distribution plots of the stress component differences. Shear stress differences determined by the model lie within the range $-220 \mathrm{MPa}$ to $+150 \mathrm{MPa}$ with the extremes in close proximity with grain boundaries and triple junctions. The residual shear stress differences based on the average mean stress in each grain show a narrow distribution of stress difference with high frequency between $+/-50 \mathrm{MPa}$ as shown in Fig. 8(b). The overall distributions of both shear and direct stress differences show very good agreement with the experimental results shown in Fig. 4. Both the polycrystal model and HREBSD measurements show a much higher frequency of high shear stress difference compared to direct stress components, and that the $\mathrm{xx}$ and yy stress distributions are similar to one another. The range of stresses measured ( -300 to $+300 \mathrm{MPa})$ using EBSD is larger than that predicted by the model. It has been pointed out $[10,24]$ that the HR-EBSD technique is capable of picking up locally high 
stresses generated through discrete dislocation structure formation which is not captured by the continuum dislocation model.

The predicted frequency distributions of corresponding hydrostatic and effective stress differences are similarly calculated using Eqs. 10 and 12 and are shown in Fig. 9(a). The crystal plasticity predicted distributions across the microstructure show strong similarity to those obtained from experiments shown in Fig. 5(a), where the effective stress difference also shows a negatively skewed normal distribution. The skewed peak in the experiments is found to be about $-100 \mathrm{MPa}$ whereas that predicted is $\sim 50 \mathrm{MPa}$. The range of effective and hydrostatic stresses is found to be broader from the EBSD measurements than that from the model, but in other respects, the distributions map very similar behaviour.

(a)

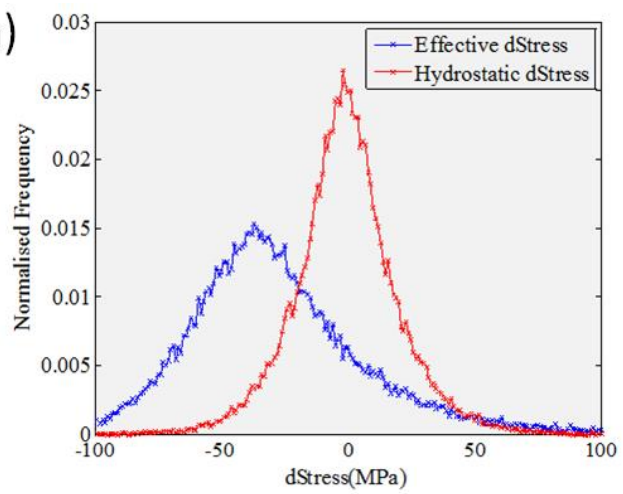

(c)

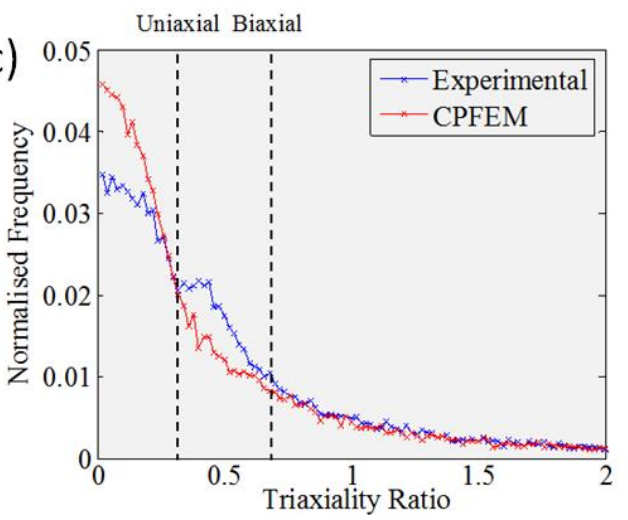

(b)

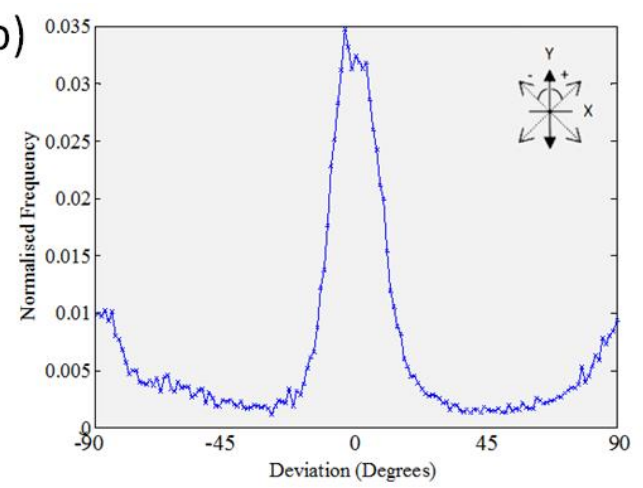

(d)

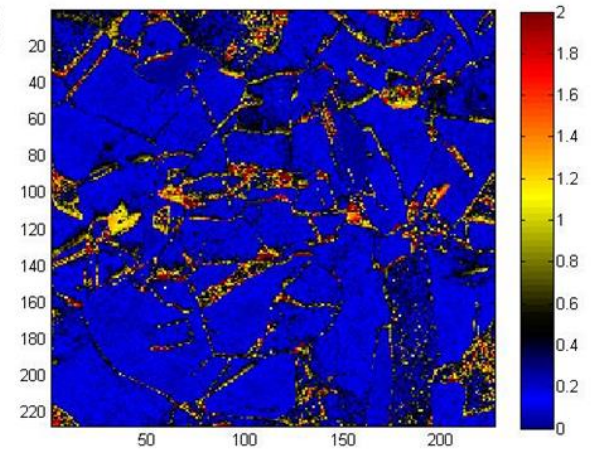

Fig. 9. Predicted frequency distributions of (a) hydrostatic and effective stress differences, (b) angular deviation between the remote load direction and local microstructural principal stress direction, (c) predicted and HREBSD measured distribution of triaxiality ratio within the polycrystal microstructure, and (d) EBSD-measured stress triaxiality ratio distribution in the experimental copper sample

The distribution of the angular deviation between the local maximum principal stress direction and the remote loading direction is plotted in Fig. 9(b) where the highest frequencies of deviations lie within the $+/-45^{\circ}$ range. This may be compared with the equivalent experimental results shown in Fig. 5(b) for which the distribution is similar but somewhat broader; that is, there exist higher numbers of microstructure locations in the experimental sample than for the model polycrystal for which bigger 
deviations exist. This indicates the experimental microstructure contains features which increase the local heterogeneity more so than that for the polycrystal model and may well result from annealing twins as discussed earlier, as well as from the general propensity for the HR-EBSD technique to capture higher, localised stresses for non-continuum reasons. Interestingly, the model predicted angular deviations show tails of higher frequency of $90^{\circ}$ deviations than do the EBSD measurements.

The predicted polycrystal distribution of the stress triaxiality defined in Eqn. 13 is shown in Fig. 9(c) together with that from the EBSD measurements, showing good agreement. However, there is greater frequency of stress triaxiality of 0.5 (indicating a stress state between uniaxial and biaxial) observed experimentally than for the crystal model together with a reversal giving higher frequency of very low triaxialities from the model. The former could well result from irregular grain morphology and more complex microstructural features (e.g. annealing twins) not incorporated in the model. Fig. 9(d) illustrates the experimentally measured stress triaxiality ratio distribution, which indicates that triaxiality ratios of around 0.5 (shown by the black contours) are found along and close to microstructural interfaces and boundaries potentially explaining the differences in the frequencies from model and measurement shown in Fig. 9(c). This suggests that detailed characterisation of gain boundaries and interfaces could benefit the model prediction of polycrystal behaviour. However, the simple hexagonal mapped polycrystal model with representative texture gives exceptionally good agreement of stress triaxiality distribution within the microstructure when compared with experimental measurement.

It is notable that the quality of agreement is obtained despite the pseudo three-dimensional nature of the model polycrystal representation in which just a single layer of grains is explicitly modelled. The key representation in the crystal model is that of texture in order to faithfully capture the experimentally determined textures (given in Figs. 6 and 3 respectively). As discussed earlier, the detail of the experimental grain morphology and twin features have not been represented in the model, nor has the subsurface microstructure. This therefore suggests that the statistical distributions of the microstructure-level stresses (including effective, hydrostatic and triaxiality quantities) are dominated by texture effects as opposed to morphology, and this is why the crystal model captures well the experimentally measured stress states. The stress is driven by the elastic modulus, which in turn because of its intrinsic crystal anisotropy, leads to strong micro-textural effects on stress. This, it is argued, explains the key role of texture in crystal modelling.

\subsection{Assessment of microstructurally-sensitive and global Schmid factors and resolved shear stresses}

We next utilise the polycrystal model to address Schmid analysis in the model Cu polycrystal under conditions of loading both at the point of first macroscopic yield and that at $1 \%$ and $6 \%$ strain. Our 
interest is again that of the global and local-calculated Schmid factors and slip system resolved shear stresses. The polycrystal model shown in Fig. 7 is subject to uniaxial straining.

At the point of first macro yield at a strain of $0.13 \%$, and utilising Eqs. 5 and 6 , the local $\left(\mathrm{SF}^{\mu}\right)$ and global $\left(\mathrm{SF}^{\mathrm{m}}\right)$ Schmid factors are extracted and their frequency distributions plotted across the polycrystal model shown in Fig. 10(a). At this point in the loading (at first yield), the local and globaldetermined Schmid factor distributions show fairly close agreement. However, notably there are differences in that the frequency of the common Schmid factors is lower when account is taken of local grain constraint effects, or conversely, the frequency of global, macroscale Schmid factors is over-predicted. Taking due account of the local stress state generated by microstructural constraint, it is seen that variations of Schmid factor within a single grain occur, contrary to the global Schmid factor analysis as shown in Fig. 10(b).

(a)

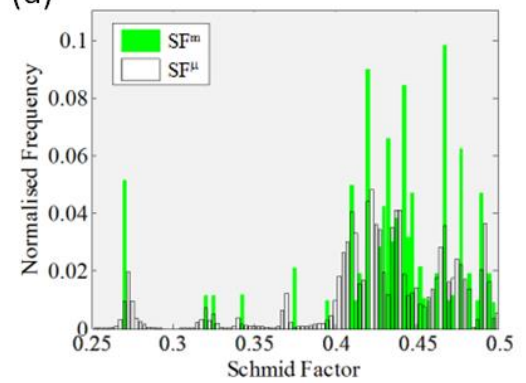

(b)

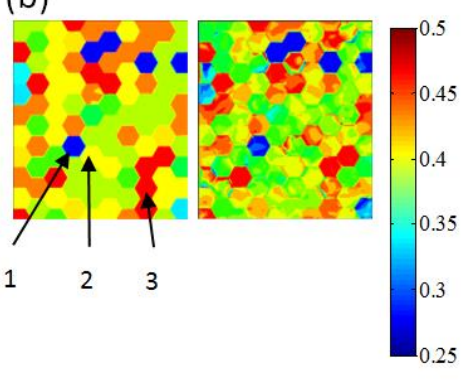

(c)

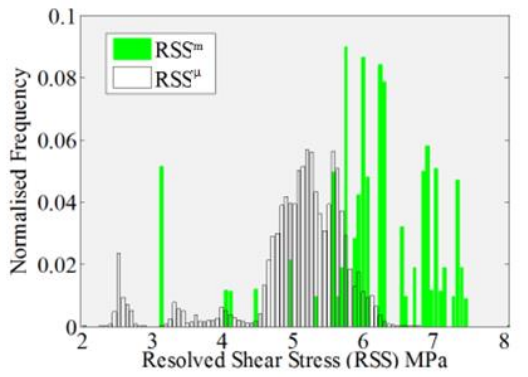

Fig. 10 - Model polycrystal behaviour at point of first yield showing (a) distributions of global $\left(\mathrm{SF}^{\mathrm{m}}\right)$ and local $\left(\mathrm{SF}^{\mu}\right)$ Schmid factors, (b) spatial distributions of (left) global $\left(\mathrm{SF}^{\mathrm{m}}\right)$ and (right) local, (SF$\left.{ }^{\mu}\right)$ Schmid factors, and (c) global and local determined resolved shear stresses

The global $\left(\mathrm{RSS}^{\mathrm{m}}\right)$ and local $\left(\mathrm{RSS}^{\mu}\right)$ resolved shear stresses (from Eqns. 7 and 8 respectively) are also compared within the polycrystal at the point of yielding, and their frequency distributions are shown in Fig. 10(c). Here, revealing that the macro/global Schmid factor approach significantly overestimates the local frequencies of high resolved shear stresses and therefore likely slip activity compared to the locally calculated resolved shear stress.

The same analysis is next carried out for the model copper polycrystal but at applied strains of $1 \%$, shown in Fig. 11(a) and (b), and at the peak strain of 6\%, in Fig. 11(c) and (d). At 1\% deformation, the lowest global peak Schmid factor of 0.27 is not observed at all when considering the local model (Fig. 11(a)) and most Schmid factors are found to lie between 0.4 to 0.5 . 
(a)

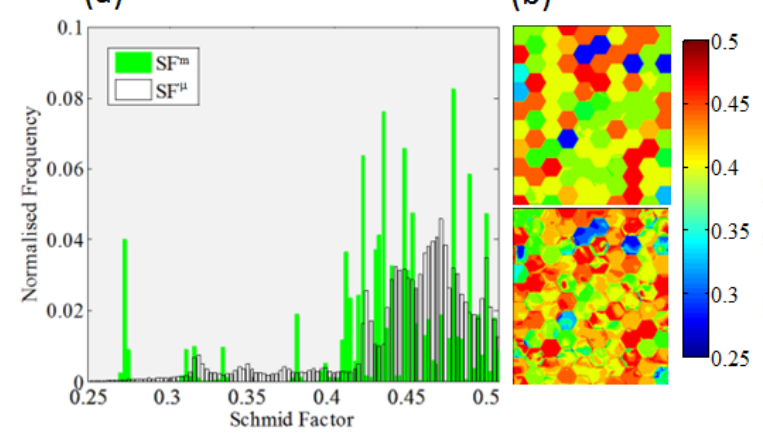

(c)

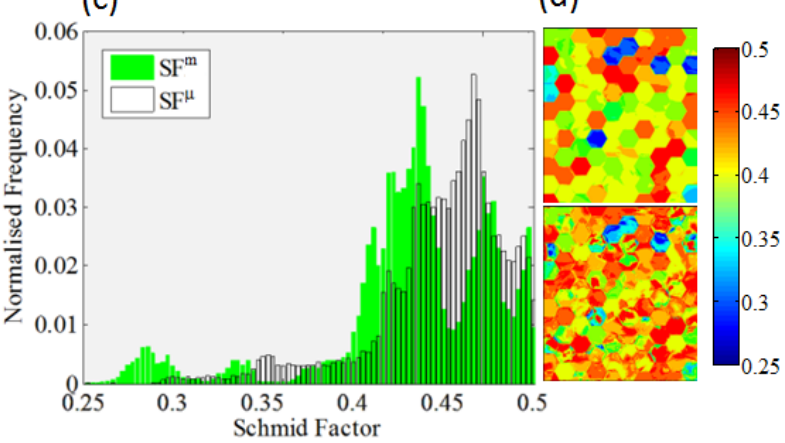

Fig. 11. (a) Local and global-determined Schmid factors at $1 \%$ applied strain, (b) corresponding spatial variation of (top) global and (bottom) local Schmid factors, (c) local and global-determined Schmid factors at 6\% applied strain, and (d) corresponding spatial variation of (top) global and (bottom) local Schmid factors

Table 2 - Development of macro-level and grain-averaged local Schmid factor for the grains identified in Fig. 10(b) (black arrows) for the applied strains shown

\begin{tabular}{|c|c|c|c|c|c|c|c|c|c|c|c|c|}
\hline & \multicolumn{4}{|c|}{1} & \multicolumn{4}{|c|}{2} & \multicolumn{4}{|c|}{3} \\
\hline Strain & $\mathrm{SF}^{\mathrm{m} \text {,avg }}$ & $\mathrm{SF}^{\mu, \max }$ & $\mathrm{SF}^{\mu, \min }$ & $\mathrm{SF}^{\mu, \text { avg }}$ & $\mathrm{SF}^{\mathrm{m} \text {,avg }}$ & $\mathrm{SF}^{\mu, \max }$ & $\mathrm{SF}^{\mu, \min }$ & $\mathrm{SF}^{\mu, \text { avg }}$ & $\mathrm{SF}^{\mathrm{m} \text {,avg }}$ & $\mathrm{SF}^{\mu, \max }$ & $\mathrm{SF}^{\mu, \min }$ & $\mathrm{SF}^{\mu, \mathrm{avg}}$ \\
\hline $\begin{array}{l}\text { Yield } \\
\text { point }\end{array}$ & 0.278 & 0.309 & 0.277 & 0.278 & 0.415 & 0.413 & 0.373 & 0.401 & 0.469 & 0.494 & 0.388 & 0.469 \\
\hline $1 \%$ & 0.278 & 0.363 & 0.285 & 0.318 & 0.416 & 0.425 & 0.366 & 0.404 & 0.469 & 0.477 & 0.428 & 0.433 \\
\hline $6 \%$ & 0.278 & 0.473 & 0.334 & 0.349 & 0.417 & 0.443 & 0.361 & 0.415 & 0.469 & 0.471 & 0.431 & 0.432 \\
\hline
\end{tabular}

At 6\% defomation, the global Schmid factor analysis indicates the presence of five hard, slip-resistent grains with Schimd values of 0.25-0.30. However the locally-determined Schmid factors show very little indication that these hard grains continue to remain slip resistent (Fig. 11(d)); rather, the heterogeneous stress states developed locally facilitate slip of other systems, generating more uniform slip fields. The macro Schmid factors for the identified grains (with black arrows in Fig. 10(b)) are calculated and compared with the grain-average local Schmid factor for that grain. The results in Table 2 show the averaged macro-level Schmid factor $\mathrm{SF}^{\mathrm{m}}$, and the grain-averaged local Schmid factors $\mathrm{SF}^{\mu}$ at strain levels corresponding to first yield, $1 \%$ and $6 \%$ respectively. At first yield, the grain-averaged $\mathrm{SF}^{\mu}$ is identical to the macro-level $\mathrm{SF}^{\mathrm{m}}$ with the exception of grain no. 2. Beyond yield, the grain-averaged local $\mathrm{SF}^{\mu}$ increases in grains 1 and 2 under $6 \%$ strain, but decreases in grain 3, indicating that the effects of local inhomogeneity have an increasing effect on local Schmid factor with increasing strain. The local maximum and minimum $\mathrm{SF}^{\mu}$ documented in Table 2 also illustrate the Schmid factor range within the grains is found to vary from 0.032 to 0.139 . Overall, as the strain increases, so the global Schmid calculation shows a more similar frequency distribution but interestingly transposed to lower Schmid factor values when compared to the more rigorous local 
Schmid analysis. Considerable differences do, however, remain demonstrating the need for caution when utilising global Schmid factors to predict activation or otherwise of slip systems within the complexity of a typical polycrystal.

\section{Microstructure-sensitive fatigue crack nucleation}

Hotspots for preferential sites of fatigue crack nucleation in a ferritic steel were addressed previously based on a new stored energy criterion proposed in [7] and integrated within a crystal plasticity modelling formulation. The introduction of a critical stored energy and a length-scale measure enabled both the locations of crack nucleation and the number of loading cycles to develop crack nucleation to be predicted for a range of experimental microstructurally-differing, polycrystal ferritic steel samples. In the present work, the stored energy criterion for fatigue crack nucleation is assessed in the context of the copper polycrystal introduced above in order to consider the relevance or otherwise of the key microstructural features leading to high Schmid factors, stress levels and (triaxial) stress states.

The stored energy criterion [7] established to locate the hotspots of fatigue crack nucleation and calculated microstructurally point-wise within a crystal plasticity formulation equates a stored energy density rate with a (Griffith-like) critical value for defect nucleation as follows

$$
\dot{G}=\frac{\dot{U} \Delta V_{s}}{\Delta A_{\mathrm{s}}}=\oint_{C} \frac{\xi \boldsymbol{\sigma}: d \boldsymbol{\varepsilon}^{p}}{\sqrt{\left(\rho_{S S D}+\rho_{G N D}\right)}} .
$$

The criterion is developed from consideration of local slip activity and the local storage volume $\Delta V_{s}$ resulting from geometrically necessary $\rho_{G N D}$ and statistically stored dislocation $\rho_{S S D}$ accumulation, defining a mean free distance induced from the plastic behaviour. The local stored energy per cycle considered is effectively derived from the point-wise crystal accumulated slip and the plastic energy associated with a stored fraction $\xi$ (assigned a value of 0.05) [7]. 


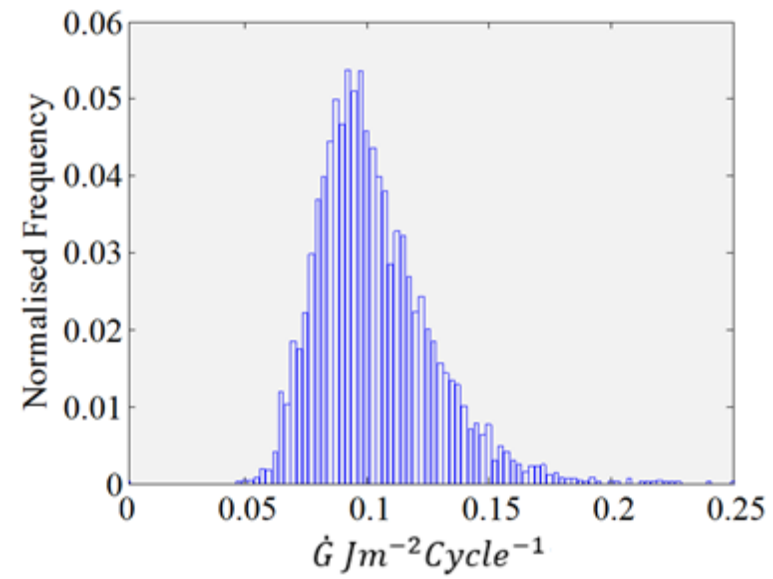

Fig. 12 - A frequency distribution of the stored energy rate for fatigue crack nucleation across the microstructural field observed at $10^{\text {th }}$ fatigue cycle of loading

The model $\mathrm{Cu}$ polycrystal is subjected to ten cycles of strain-controlled ( $2 \%$ peak strain) fatigue loading $(\mathrm{R}=0)$ with the loading and boundary conditions shown in Fig. 7. Full spatial fields of the stored energy rate are captured using the polycrystal model, and these are then assessed in the context of the Schmid factor differences and grain disorientations. Fig. 12 shows a quantitative frequency distribution of the stored energy per cycle across the copper polycrystal microstructure, showing a range of stored energy rate between $0.05-0.24 \mathrm{Jm}^{-2}$ per cycle.

After 10 fatigue cycles, slip accumulation clearly occurs as shown in Fig. 13(a). Thus there are localised establishments of stored energy associated with the plastic deformation, and generally, the stored energy rate in most regions across the model microstructure is $\sim 0.1 \mathrm{Jm}^{-2}$ per cycle. The focus for fatigue crack nucleation hotspots is argued to be that for which higher stored energy rates per cycle are observed. These twelve locations, (the highest $1.5 \%$ across the polycrystal, corresponding to $0.153 \mathrm{Jm}^{-2}$ or higher) are indicated in the microstructure as shown in Fig. 13(b), and an overlay of the grain boundaries across the microstructure is shown on Fig. 13(c).

(a)
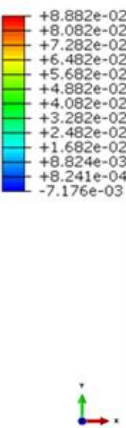

(b)

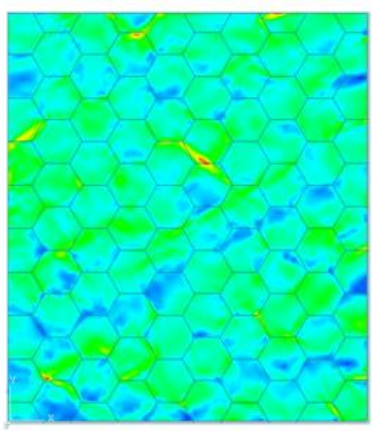

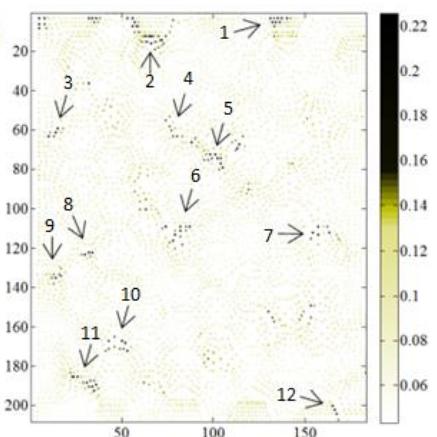

(c)

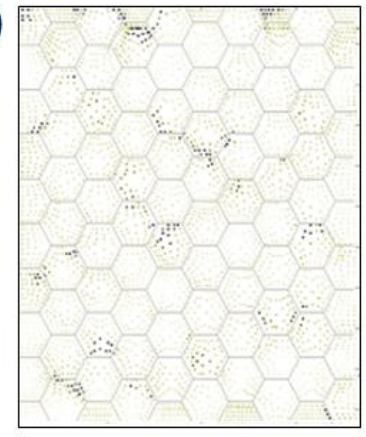

Fig. 13 - Spatial distribution at the $10^{\text {th }}$ fatigue cycle of (a) effective plastic strain, (b) stored energy rate per cycle $(\dot{G})$, and (c) as (b) but with grain boundary overlay 
Table 3 - Identification of fatigue crack nucleation hotspots within the polycrystal model.

\begin{tabular}{|c|c|c|c|c|}
\hline Hotspot & Type & $\begin{array}{c}\text { Frequency of } \\
\text { hotspots } \\
\text { (strength) }\end{array}$ & $\begin{array}{c}\text { Schmid factor } \\
\text { differences }\end{array}$ & $\begin{array}{c}\text { Average } \\
\text { disorientations } \\
\text { (Degree) }\end{array}$ \\
\hline 1 & GB & 9 & 0.083 & 57.2 \\
\hline 2 & GB & 12 & -0.066 & 24.8 \\
\hline 3 & TJ & 4 & $-0.107,0.099$ & 63.2 \\
\hline 4 & GB & 2 & 0.026 & 49.1 \\
\hline 5 & GB & 11 & 0.096 & 68.6 \\
\hline 6 & GB & 8 & 0.019 & 77.2 \\
\hline 7 & GB & 5 & 0.091 & 61.2 \\
\hline 8 & TJ & 3 & $0.046,0.047$ & 62.6 \\
\hline 9 & TJ & 2 & $-0.051,0.007$ & 60.3 \\
\hline 10 & GB-G & 7 & 0.083 & 36.4 \\
\hline 11 & GB & 9 & -0.002 & 48.5 \\
\hline 12 & GB & 4 & 0.097 & 36.0 \\
\hline
\end{tabular}

Table 3 shows a summary of the features of each of the twelve hotspots so identified, indicating relevant microstructural features, crystal orientations and strength of hotspot. The hotspot strength is determined simply by the number of occurrences of high stored energy rate identified at the fatigue crack nucleation hotspot. The Schmid factor differences are calculated between the neighbouring grains local to the hotspots where the negative Schmid factor difference indicates the hotspot in a harder grain. The disorientation angle is also considered to understand any hotspot characteristics and is simply the orientation difference between two neighbouring failure grains. This is calculated with knowledge of the crystallographic orientations of the two neighbouring grains and is well documented $[37,38]$.

From Table 3, the most common type of hotspot identified occurs at or close to a grain boundary (GB), largely resulting from a high rate of slip accumulation as shown in Fig. 13(a). The Schmid factor differences across the grain boundary hotspots (i.e. over the grain containing the nucleation hotspots and the neighbouring grain) show variation from -0.066 to 0.096 where both extreme values correspond to strongest sites for predicted nucleation. As shown in Fig. 14(a), Schmid factor values along a path from the nucleation hotspot grain (location 1) to the grain boundary (location 3 ) and into the neighbouring grain (location 5) are plotted. Only grain boundary type nucleation hotspots are included and show that the majority, with exception of hotspot No. 2 and 11, have a higher Schmid factor value at location 1 which tends to decrease towards location 5 . This suggests softer grains are favoured sites of nucleation rather than the neighbouring harder grains.

i - Type: (GB) grain boundary only, (GB-G) grain boundary and within corresponding grain, (TJ) triple junctions only 

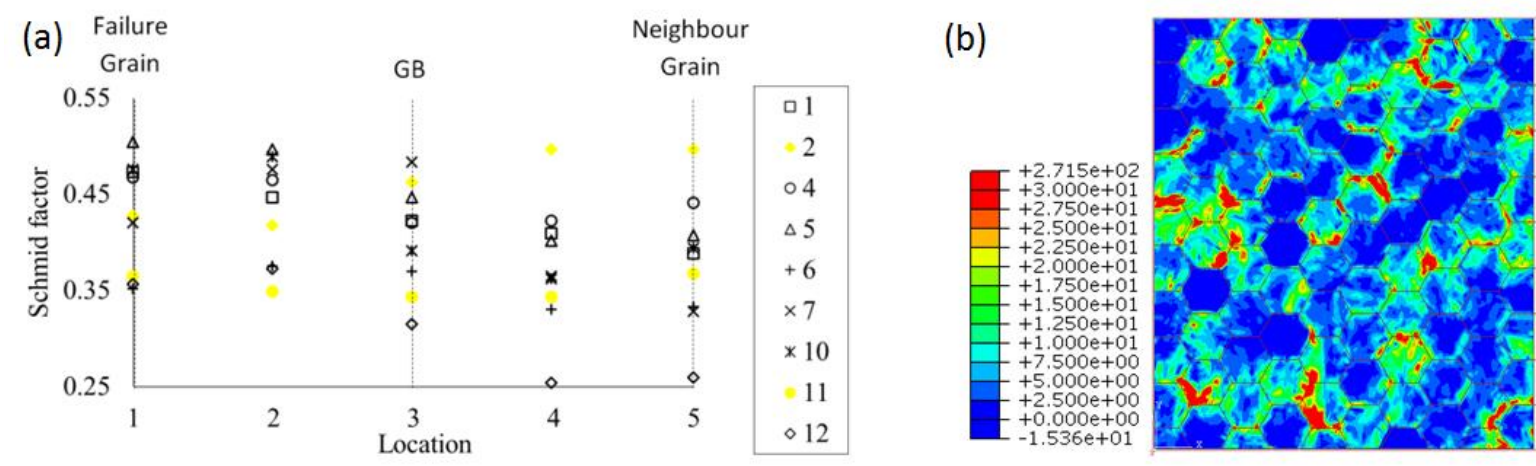

Fig. 14 - (a) Schmid factor distribution across grain boundary (GB) nucleation hotspots across RVE copper polycrystal (location 1 to 5 is path ID from nucleation hotspot to neighbouring grain) and (b) geometrically necessary dislocation distribution (units in $\times 10^{12} \mathrm{~m}^{-2}$ )

GND distributions also show that harder grains typically generate higher localisation of GND densities around the grain to accommodate the lattice curvature generated by plastic strain gradients, as shown in Fig. 14(b). Of the 12 nucleation hotpots, with the exception of hotspots 4 and 10, they all lie within regions of high GND density greater than $1 \times 10^{14} \mathrm{~m}^{-2}$.

The importance of these results is expanded upon by a further set of ten simulations utilising the same model RVE polycrystal but for which differing random realisations of the texture have been established from the set of orientations in the initial study above, to provide a sound statistical basis for further examination of fatigue crack nucleation hotspots. In recent cyclic fatigue studies [39], the point-wise evolution of stored energy has been demonstrated to stabilise and achieve steady-state evolution after a few loading cycles so that in order to identify stored energy hotspots, it is not unreasonable to investigate a series of monotonically loaded polycrystals for the purposes of establishing sufficient polycrystal analyses to be statistically representative. Experimental investigations of accumulated slip at the microstructure-sensitive level also show that the form of the distribution has already been established within the first loading cycle and that subsequent cycles tend to increase the intensity of the localisations through, for example, progressive ratcheting processes [6]. 


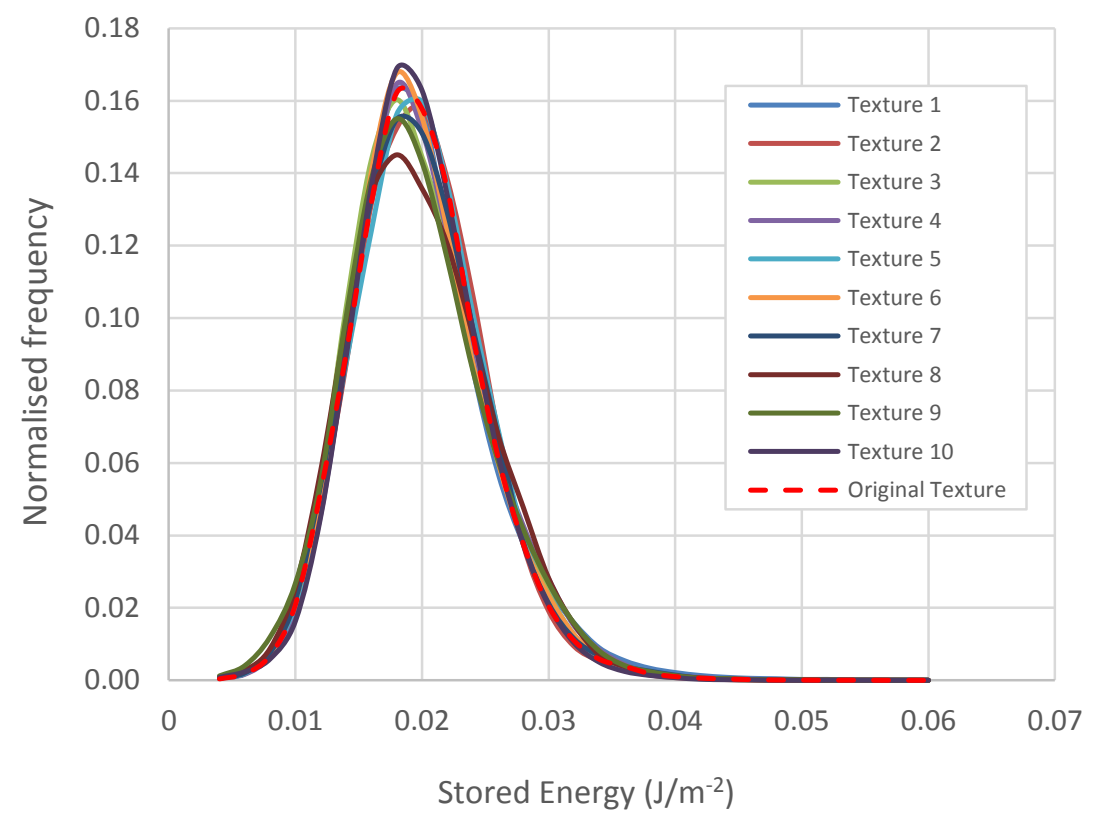

Fig. 15 - Comparison of the total number of hotspot types for each texture variation by category: triple junction (TJ), grain boundary (GB), and interior to the grain (GB-G)

In a similar manner to Fig. 12, the normalised distribution of the stored energy across each polycrystal, at the end of the loading, for each realisation, is presented in Fig. 15. In this set of studies, the stored energy rate of Eqn. 19 is integrated using a forward Euler scheme to calculate the cumulative stored energy at the end of each loading history. The variation of normalised frequency suggests that simple variation of textural realisation in the polycrystal has a direct impact on the stored energy accumulation due to the inter-granular interactions of harder and softer grains (in terms of local Schmid factors).

Fig. 16 shows a histogram of the various categories of hotspot types and their frequencies, for each texture realisation, including that studied earlier and labelled original texture. In this statistical study, a hotspot is defined as any point reaching a specific threshold value of stored energy, namely 0.036 $\mathrm{J} / \mathrm{m}^{-2}$. This threshold value is chosen since it gives approximately the top $1.5 \%$ of stored energies in each polycrystal realisation. Specific geometrical markers defining the occurrences of GB, TJ, and GB-G locations have been rigorously determined and are appropriate as the same geometrical grain model was used in each study. 


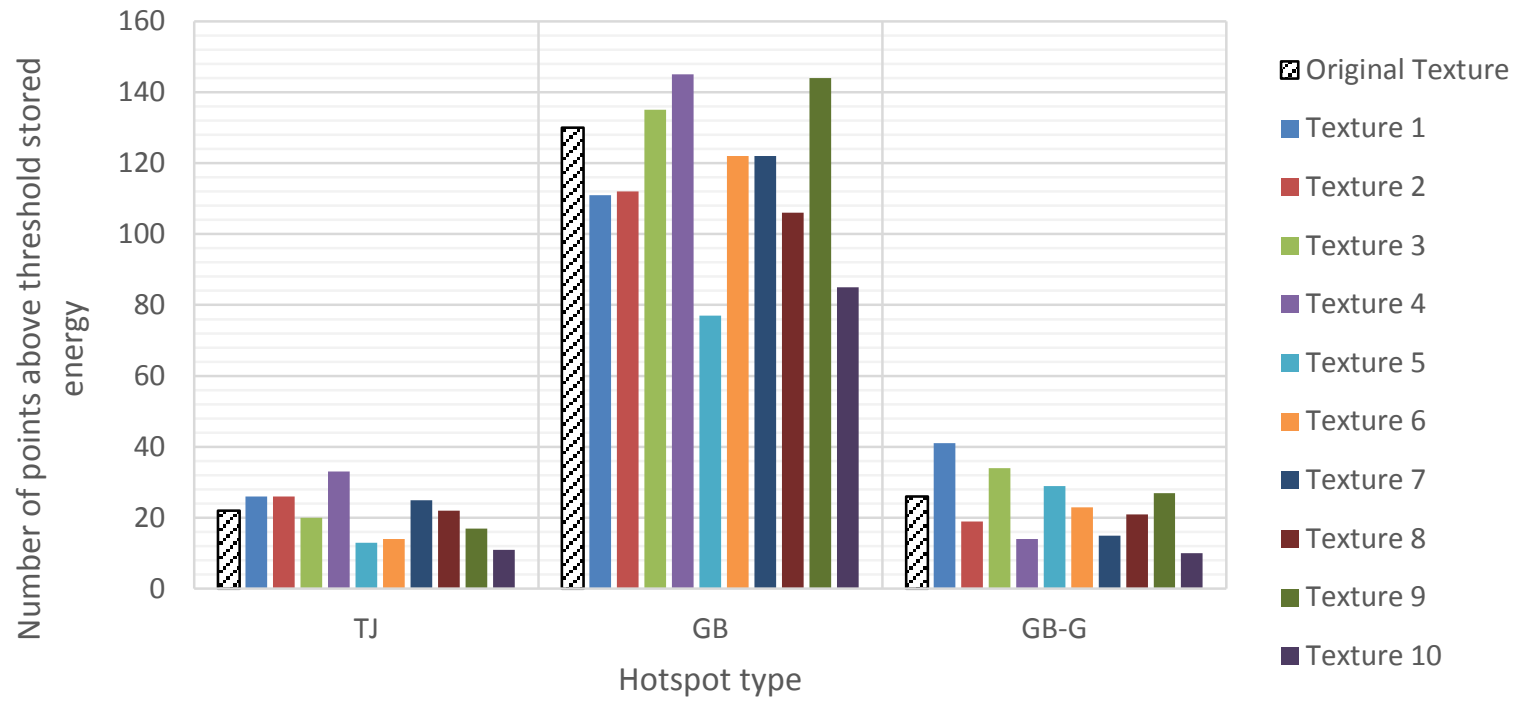

Fig. 16 - Comparison of the total number of hotspot types for each texture realisation by category, triple junction (TJ), grain boundary (GB), and interior to the grain (GB-G)

Grain boundary locations dominate the hotspots for stored energy. For each realisation of the texture, instances of GB hotspots far exceed either TJ or GB-G points, supporting the argument that grain boundaries are the most likely locations for fatigue crack nucleation sites.

Hence the stored energy criterion for nucleation previously shown to be able to rank and capture the location of fatigue crack nucleation and the loading cycles necessary to cause it [7], indicates that key microstructural features associated with crack nucleation are grain boundaries (as opposed to triple junctions). The magnitudes of Schmid factor and disorientation differences between soft and hard grains with respect to indicated nucleation sites show no strong correlation. Microstructure hotspots in general show that those locations giving rise to the highest stored energies (and by implication, crack nucleation) are those in grains which are better orientated for slip (softer grains) than their neighbouring (hard) grains. Recent studies [11, 40, 41] have addressed characterising grain boundaries to better identify the roles of the interfaces in the development of the local slip mechanisms, and micro-crack nucleation. However, the understanding of the character of grain boundaries for local slip transfer and its implementation into CPFE remains a work in progress, but there is little doubt that it is likely to be of importance in the context of localised crack nucleation phenomena.

\section{Conclusion}

Residual stresses measured from experimental HR-EBSD data for an OFHC copper polycrystal under $6 \%$ deformation develop broad distributions of stress, and stress state including triaxiality. Both the experimental and the calculated stress triaxialities are found in the majority to be representative of those under uniaxial towards biaxial stress states. However, a significant tail of higher triaxial stress 
states are found to exist, potentially indicative of defect nucleation sites. The stress components were found to be distributed approximately normally.

Further, an RVE microstructural crystal plasticity model with gradient-enhanced crystal plasticity with comparable texture reproduced the same generic microstructural stress distributions showing excellent agreement with experimental measurements particularly for the distribution of stress triaxiality. Global and local calculations of Schmid factors are compared using the CPFE model at the point of yielding (smaller deformation) and after subsequent straining. The Schmid factors with high frequency were found to be the about the same irrespective of method of calculation, but the local variation of intragranular Schmid factor were not. It was also found that the magnitude of globallycalculated resolved shear stresses were generally much higher than those determined rigorously using local, grain-level stress information, therefore overestimating potential slip activity within the microstructure. Under larger strains, the differences between local and globally calculated Schmid factors increase.

A stored energy criterion for fatigue crack nucleation indicates that preferential sites for fatigue crack nucleation are local to grain boundaries (as opposed to triple junctions), and that hard-soft grain interfaces where high GND densities develop are preferable.

\section{Acknowledgements}

This work is part of a Collaborative R\&T Project, SILOET II, Project 10, Virtual Engine Design System, supported by the Technology Strategy Board and carried out by Rolls-Royce plc and Imperial College London. The authors would like to acknowledge helpful discussions with Dr. B. Lan and Dr. T.B. Britton. FPED acknowledges his Royal Academy of Engineering/Rolls-Royce research chair funding.

\section{References}

[1] P.D. Littlewood, A.J. Wilkinson, Local deformation patterns in Ti-6Al-4V under tensile, fatigue and dwell fatigue loading, International Journal of Fatigue, 43 (2012) 111-119.

[2] P.D. Littlewood, T.B. Britton, A.J. Wilkinson, Geometrically necessary dislocation density distributions in Ti-6Al-4V deformed in tension, Acta Materialia, 59 (2011) 6489-6500.

[3] J.D. Carroll, W. Abuzaid, J. Lambros, H. Sehitoglu, High resolution digital image correlation measurements of strain accumulation in fatigue crack growth, International Journal of Fatigue, 57 (2013) 140-150.

[4] T.B. Britton, J. Jiang, P. Karamched, A. Wilkinson, Probing Deformation and Revealing Microstructural Mechanisms with Cross-Correlation-Based, High-Resolution Electron Backscatter Diffraction, Jom, 65 (2013) 1245-1253.

[5] G.I. Taylor, Analysis of plastic strain in a cubic crystal, Stephen Timoshenko 60th Anniversary Volume, (1938) 218-224.

[6] T. Zhang, J. Jiang, B.A. Shollock, T.B. Britton, F.P. Dunne, Slip localization and fatigue crack nucleation near a non-metallic inclusion in polycrystalline nickel-based superalloy, Materials Science and Engineering: A, 641 (2015) 328-339. 
[7] V.V.C. Wan, D.W. MacLachlan, F.P.E. Dunne, A stored energy criterion for fatigue crack nucleation in polycrystals, International Journal of Fatigue, 68 (2014) 90-102.

[8] F.P.E. Dunne, A.J. Wilkinson, R. Allen, Experimental and computational studies of low cycle fatigue crack nucleation in a polycrystal, International Journal of Plasticity, 23 (2007) 273-295.

[9] C. Madrigal, F.P.E. Dunne, M.R. Bache, Experimental and crystal plasticity studies of deformation and crack nucleation in a titanium alloy, The Journal of Strain Analysis for Engineering Design, 45 (2010) 391-399.

[10] J. Jiang, T.B. Britton, A.J. Wilkinson, Mapping type III intragranular residual stress distributions in deformed copper polycrystals, Acta Materialia, 61 (2013) 5895-5904.

[11] J. Jiang, T.B. Britton, A.J. Wilkinson, Evolution of intragranular stresses and dislocation densities during cyclic deformation of polycrystalline copper, Acta Materialia, 94 (2015) 193-204.

[12] S. Sriram, D.M. Dimiduk, P.M. Hazzledine, V.K. Vasudevan, The geometry and nature of pinning points of $\frac{1}{2} \quad$ 〈110] unit dislocations in binary TiAl alloys, Philosophical Magazine A, 76 (1997) 965-993. [13] B. Viguier, M. Cieslar, K. Hemker, J. Martin, J. Horton, I. Baker, S. Hanada, R. Noebe, D. Schwartz, High-Temperature Ordered Intermetallic Alloys VI, in: Materials Research Society Symposium Proceedings, 1995, pp. 653.

[14] J. Kacher, B.P. Eftink, B. Cui, I.M. Robertson, Dislocation interactions with grain boundaries, Current Opinion in Solid State and Materials Science, 18 (2014) 227-243.

[15] L. Wang, Y. Yang, P. Eisenlohr, T.R. Bieler, M.A. Crimp, D.E. Mason, Twin Nucleation by Slip Transfer across Grain Boundaries in Commercial Purity Titanium, Metallurgical and Materials Transactions A, 41 (2009) 421-430.

[16] T.R. Bieler, P. Eisenlohr, C. Zhang, H.J. Phukan, M.A. Crimp, Grain boundaries and interfaces in slip transfer, Current Opinion in Solid State and Materials Science, 18 (2014) 212-226.

[17] M.D. McMurtrey, G.S. Was, B. Cui, I. Robertson, L. Smith, D. Farkas, Strain localization at dislocation channel-grain boundary intersections in irradiated stainless steel, International Journal of Plasticity, 56 (2014) 219-231.

[18] H. Lim, J.D. Carroll, C.C. Battaile, T.E. Buchheit, B.L. Boyce, C.R. Weinberger, Grain-scale experimental validation of crystal plasticity finite element simulations of tantalum oligocrystals, International Journal of Plasticity, 60 (2014) 1-18.

[19] S. Birosca, F. Di Gioacchino, S. Stekovic, M. Hardy, A quantitative approach to study the effect of local texture and heterogeneous plastic strain on the deformation micromechanism in RR1000 nickel-based superalloy, Acta Materialia, 74 (2014) 110-124.

[20] E. Mikkola, G. Marquis, J. Solin, Mesoscale modelling of crack nucleation from defects in steel, International Journal of Fatigue, 41 (2012) 64-71.

[21] T.R. Bieler, P. Eisenlohr, F. Roters, D. Kumar, D.E. Mason, M.A. Crimp, D. Raabe, The role of heterogeneous deformation on damage nucleation at grain boundaries in single phase metals, International Journal of Plasticity, 25 (2009) 1655-1683.

[22] M. Risbet, X. Feaugas, C. Guillemerneel, M. Clavel, Damage in nickel base superalloy: Influence of local parameters measured by electron backscattered diffraction and atomic force microscopy, Scripta Materialia, 60 (2009) 269-272.

[23] N.L. Phung, V. Favier, N. Ranc, Evaluating Schmid criterion for predicting preferential locations of persistent slip markings obtained after very high cycle fatigue for polycrystalline pure copper, International Journal of Fatigue, 77 (2015) 115-127.

[24] J. Jiang, T. Benjamin Britton, A.J. Wilkinson, Evolution of intragranular stresses and dislocation densities during cyclic deformation of polycrystalline copper, Acta Materialia, 94 (2015) 193-204.

[25] B. Larrouy, P. Villechaise, J. Cormier, O. Berteaux, Grain boundary-slip bands interactions: Impact on the fatigue crack initiation in a polycrystalline forged Ni-based superalloy, Acta Materialia, 99 (2015) 325-336.

[26] K. Zhang, K. Yang, A. Huang, X. Wu, C. Davies, Fatigue crack initiation in as forged Ti-6Al-4V bars with macrozones present, International Journal of Fatigue, 80 (2015) 288-297. 
[27] M.D. Sangid, H.J. Maier, H. Sehitoglu, A physically based fatigue model for prediction of crack initiation from persistent slip bands in polycrystals, Acta Materialia, 59 (2011) 328-341.

[28] D. Hull, D.J. Bacon, Chapter 3 - Movement of Dislocations, in: D. Hull, D.J. Bacon (Eds.) Introduction to Dislocations (Fifth Edition), Butterworth-Heinemann, Oxford, 2011, pp. 43-62.

[29] T. Zhang, D.M. Collins, F.P. Dunne, B.A. Shollock, Crystal plasticity and high-resolution electron backscatter diffraction analysis of full-field polycrystal $\mathrm{Ni}$ superalloy strains and rotations under thermal loading, Acta Materialia, 80 (2014) 25-38.

[30] G. Trattnig, T. Antretter, R. Pippan, Fracture of austenitic steel subject to a wide range of stress triaxiality ratios and crack deformation modes, Engineering Fracture Mechanics, 75 (2008) 223-235.

[31] A.J. Wilkinson, G. Meaden, D.J. Dingley, High-resolution elastic strain measurement from electron backscatter diffraction patterns: New levels of sensitivity, Ultramicroscopy, 106 (2006) 307313.

[32] T.B. Britton, A.J. Wilkinson, High resolution electron backscatter diffraction measurements of elastic strain variations in the presence of larger lattice rotations, Ultramicroscopy, 114 (2012) 82-95. [33] T.B. Britton, J. Jiang, R. Clough, E. Tarleton, A.I. Kirkland, A.J. Wilkinson, Assessing the precision of strain measurements using electron backscatter diffraction - part 1: Detector assessment, Ultramicroscopy, 135 (2013) 126-135.

[34] E.H. Lee, Elastic-Plastic Deformation at Finite Strains, Journal of Applied Mechanics, 36 (1969) 16.

[35] C.A. Sweeney, W. Vorster, S.B. Leen, E. Sakurada, P.E. McHugh, F.P.E. Dunne, The role of elastic anisotropy, length scale and crystallographic slip in fatigue crack nucleation, Journal of the Mechanics and Physics of Solids, 61 (2013) 1224-1240.

[36] F.P.E. Dunne, D. Rugg, A. Walker, Lengthscale-dependent, elastically anisotropic, physicallybased hcp crystal plasticity: Application to cold-dwell fatigue in Ti alloys, International Journal of Plasticity, 23 (2007) 1061-1083.

[37] V. Randle, O. Engler, Introduction to texture analysis: macrotexture, microtexture and orientation mapping, CRC Press, 2000.

[38] A.J. Schwartz, M. Kumar, B.L. Adams, D.P. Field, Electron backscatter diffraction in materials science, Springer, 2009.

[39] V. Wan, J. Jiang, D. MacLachlan, F. Dunne, Microstructure-sensitive fatigue crack nucleation in a polycrystalline Ni superalloy, International Journal of Fatigue, (2016).

[40] A.F. Knorr, M. Marx, Calculating the Resistance of a Grain Boundary against Fatigue Crack Growth, Advanced Materials Research, 891 (2014) 929-935.

[41] M. Sauzay, M.O. Moussa, Comparison between Pile-Up Singularities and Stress Fields Induced by Thin Slip Bands. Application to the Prediction of Grain Boundary Microcrack Nucleation, in: Key Engineering Materials, Trans Tech Publ, 2014, pp. 61-66. 